Original Research Paper

\title{
Oral Clindamycin as Drug of Choice for Scabies Patients with Secondary Bacterial Infections in West Java, Indonesia
}

\author{
Hendra Gunawan, Unwati Sugiri, Nurhasanah, Kristina Makarti and Oki Suwarsa \\ Departement of Dermatology and Venereology, Faculty of Medicine, \\ Universitas Padjadjaran - Dr. Hasan Sadikin Hospital, Bandung, Indonesia
}

Article history

Received: 18-10-2017

Revised: $29-12-2017$

Accepted: 09-01-2018

Corresponding Author:

Hendra Gunawan

Departement of Dermatology

and Venereology, Faculty of

Medicine, Universitas

Padjadjaran- Dr. Hasan

Sadikin Hospital, Bandung,

Indonesia

Email: h.gunawan2016@unpad.ac.id

\section{Introduction}

Bacterial skin infections or pyodermas are still common in most developing countries (Hay et al., 2014). It is a superficial skin infection that primarily affects children. Mostly, adults acquire pyoderma through close contact with infected children (Halpern and Heymann, 2008). Pyoderma is classified into primary and secondary types. One study in 2012 reported that among the various diseases that were found to be associated with primary pyodermas, scabies was the most common, seen in $8.50 \%$ cases (Gandhi et al., 2012). Most of pyodermas are caused by Gram positive bacteria,

\begin{abstract}
The aim of this study was to identify the causative secondary bacterial infection in scabies and its susceptibility against systemic antimicrobials. We performed a cross-sectional descriptive study of 34 scabies patients clinically diagnosed by investigators through consecutive sampling in one District Hospital in West Java, Indonesia from January to March 2017. The secondary bacterial infection was confirmed by Gram staining. Bacterial culture was derived from intact pustules, then identified using Vitek® 2 system, including its susceptibility against 30 systemic antimicrobials. The result of bacterial identification consisted of $48.89 \%$ Group A Beta-Hemolytic Streptococcus (GABHS), 44.44\% Staphyloccocus aureus (S. aureus), 4.44\% Staphylococcus epidermidis (S. epidermidis) and $2.22 \%$ Klebsiella pneumoniae (K. Pneumoniae). All bacterias were sensitive to carbapenem group, however resistant to cephradine and kanamycin. The overall percentages of GABHS sensitivity to the tested antibiotics were as follows: $95.45 \%$ for chloramphenicol and ceftriaxone, $90.91 \%$ for amoxicillin/clavulanate, $86.36 \%$ for clindamycin, cloxacillin, cefotaxime, $72.27 \%$ for ciprofloxacin and methicillin. Sensitivity of S. aureus to the antibiotics were as follows: $100.00 \%$ for methicillin, $95.00 \%$ for clindamycin and cloxacillin, $90.00 \%$ for ciprofloxacin and levofloxacin, $85.00 \%$ for cotrimoxazole and $75.00 \%$ for ceftriaxone. The sensitivity of $S$. epidermidis to clindamycin, amoxicillin/clavulanate and methicillin were $100.00 \%$. All of K. pneumoniae (100.00\%) were sensitive to ciprofloxacin, cotrimoxazole, ampicillin/sulbactam, cefazolin, ceftriaxone, ceftazidime and cefepime. The most common etiology of secondary infection in scabies were GABHS and $S$. aureus with varying sensitivity and oral clindamycin
\end{abstract}

Keywords: Antimicrobial Susceptibility, Secondary Infection, Scabies

especially either $S$. aureus or GABHS (Bowen et al., 2015). $S$. aureus in pyoderma may cause serious complications, such as invading the bloodstream, producing bacteremia (Vanderkoo et al., 2011) and infective endocarditis (Twele et al., 2010). Meanwhile Acute Post-Streptococcal Glomerulonephritis (APSGN) in several studies (Streeton et al., 1995; White et al., 2001; Rodriguez-Iturbe and Haas, 2016), acute rheumatic heart disease (Edison et al., 2015) and death (Wong and Stevens, 2013) may follow GABHS infections.

Brook (1995) reported that scabies was often accompanied by secondary bacterial infection. This secondary bacterial infection may develope to 
antimicrobial resistance. Bacterial resistance to antimicrobials may lead to inefficient treatment, cripple the ability to fight infectious disease and difficulty to eradicating the infection (CDCP, 2013). It is not always feasible to perform pus cultures and sensitivity tests before instituting antimicrobial therapy for pyodermas. Therefore, it becomes imperative to have periodic updates on the causative organisms, their strains and antimicrobial sensitivity patterns in any given local community. Hence, this study was undertaken to identify the causative microorganisms of secondary infection in scabies and their susceptibility againts systemic antimicrobials in one District Hospital in West Java, Indonesia on 2017.

\section{Materials and Methods}

\section{Patients}

The study was approved by the Health Research Ethics Committee, Faculty of Medicine, Universitas Padjadjaran, Bandung, West Java, Indonesia with ethical clearance. Thirty-four subjects were recruited and enrolled after informed consent was explained. Patients were further divided into two age groups 0-14 years and $\geq 14$ years. All patients of scabies with secondary bacterial infection were enrolled in this study irrespective of any concomitant disease. Scabies patients were diagnosed clinically by the investigators. Clinical criteria for diagnoses were: (1) presence of papules, vesicles, nodules, or burrows at sites of predilection, (2) night itch and (3) involvement of at least another family member or companion. Patients with two of the above criteria were included in the study. Bacterial infection in scabies was marked by pustules at sites of predilection and was proved by Gram staining with the appearance of Gram-positive or Gram-negative cocci or rods. Subjects who had used systemic or topical antimicrobial agents in the preceding two weeks were excluded from the study.

\section{Bacterial Culture and Antimicrobial Susceptibility Testing}

Only one specimen was taken from every subject. The specimens were purulent material from intact pustules, obtained with $1 \mathrm{~mL}$ spuit after cleaning the surrounding skin with alcohol swab. Gram staining was conducted on the specimens which were sent to the laboratory incubated in tryptic soy broth transport medium, then cultured on both Blood agar and MacConkey agar. The plates were incubated aerobically at $37^{\circ} \mathrm{C}$ for 18 to $24 \mathrm{~h}$. The time between specimen collection and inoculation to these agar plates never exceeded $72 \mathrm{~h}$. Identification of bacteria and antimicrobial susceptibility testing using Vitek ${ }^{\circledR} 2$ were further carried out according to manufacturer's instructions. Vitek ${ }^{\circledR} 2$ uses an antimicrobial dilution according to Clinical and Laboratory Standards Institute (CLSI) (Pincus, 2006; Shetty et al., 1998) to determine Minimal Inhibitory Concentrations (MIC). MIC results are either sensitive, intermediate, or resistant antimicrobials (Pincus, 2006).

\section{Results}

Of the 34 subjects, the number of female patients was similar to the number of male patients. Patient ages ranged from two months to 70 years old. The majority $(82.35 \%)$ were children and a majority were students (55.88\%) (Table 1$)$.

From 34 specimens, $23(67.65 \%)$ of the infections detected a single causative microorganism. GABHS accounted for $35.29 \%$ of these infection, followed by $S$. aureus (29.41\%). Eleven (32.35\%) specimens yielded mixed infection and were mostly caused by mixture of GABHS and S. aureus (26.47\%) (Table 2).

Table 1: Characteristic of subjects

\begin{tabular}{lcc}
\hline & \multicolumn{2}{l}{ Number } \\
Variable & $\mathrm{n}=34$ & $\%$ \\
\hline Gender & & \\
Female & 18 & 52,94 \\
Male & 16 & 47.06 \\
Age & & \\
Children: 0-14 years old & 28 & 82.35 \\
Teenager/adult: $\geq 14$ years old & 6 & 17.65 \\
Occupation & & \\
Student & 19 & 55.88 \\
Unemployee & 13 & 38.23 \\
Housewife & 1 & 2.94 \\
Farmer & 1 & 2.94 \\
\hline
\end{tabular}

Table 2: Bacterial Culture Result

\begin{tabular}{llr}
\hline & Number & \\
Bacterial & ---------- \\
culture result & $\mathrm{n}=34$ & \multicolumn{1}{c}{$\%$} \\
\hline GABHS & 12 & 35.29 \\
S. aureus & 10 & 29.41 \\
S. epidermidis & 1 & 2.94 \\
GABHS + S. aureus & 9 & 26.47 \\
S. aureus + S. epidermidis & 1 & 2.94 \\
GABHS + K. pneumoniae & 1 & 2.94 \\
\hline
\end{tabular}

Table 3: Isolates of Microorganism from 34 speciments

\begin{tabular}{llr}
\hline Bacterial & Number & \\
culture result & -------------- \\
\hline GABHS & $\mathrm{n}=34$ & \% \\
S. aureus & 22 & 48.89 \\
S.epidermidis & 20 & 44.44 \\
K. pneumoniae & 2 & 4.44 \\
\hline
\end{tabular}


The number of microorganisms that were isolated from 34 specimens recovered 4 isolated microorganisms. GABHS and $S$. aureus were the most common species. $S$. epidermidis recovered in two specimens and $K$. pneumoniae in one specimen (Table 3).

Sensitivity test of GABHS, $S$. aureus and $S$. epidermidis against various antimicrobials are presented in Table 4 . The highest sensitivity of these microorganisms was to imipenem and meropenem (each sensitivity $100.00 \%$ ) and resistant to cephradine and kanamycin (sensitivity $0.00 \%$ ). In addition, GABHS were resistant to doxycycline, erythromycin and azithromycin, whereas $S$. epidermidis were resistant to azithromycin.

Table 4: Antimicrobial Susceptibility Tests of GABHS, S. aureus and S. epidermidis

\begin{tabular}{|c|c|c|c|c|c|c|c|c|c|c|c|c|}
\hline \multirow[b]{3}{*}{ Antimicrobial } & \multicolumn{4}{|c|}{ GABHS $(n=22)$} & \multicolumn{4}{|c|}{ S. aureus $(\mathrm{n}=20)$} & \multicolumn{4}{|c|}{ S. epidermidis $(\mathrm{n}=2)$} \\
\hline & \multicolumn{2}{|c|}{ Sensitive } & \multicolumn{2}{|c|}{ Resistant } & \multicolumn{2}{|c|}{ Sensitive } & \multicolumn{2}{|c|}{ Resistant } & \multicolumn{2}{|c|}{ Sensitive } & \multicolumn{2}{|c|}{ Resistant } \\
\hline & $\mathrm{n}$ & $\%$ & $\mathrm{n}$ & $\%$ & $\mathrm{n}$ & $\%$ & $\mathrm{n}$ & $\%$ & $\mathrm{n}$ & $\%$ & $\mathrm{n}$ & $\%$ \\
\hline Imipenem & 22 & 100.00 & 0 & 0.00 & 20 & 100.00 & 0 & 0.00 & 2 & 100.00 & 0 & 0.00 \\
\hline Meropenem & 22 & 100.00 & 0 & 0.00 & 20 & 100.00 & 0 & 0.00 & 2 & 100.00 & 0 & 0.00 \\
\hline Ceftriaxone & 21 & 95.45 & 1 & 4.55 & 15 & 75.00 & 5 & 25.00 & 1 & 50.00 & 1 & 50.00 \\
\hline Chloramphenicol & 21 & 95.45 & 1 & 4.55 & 3 & 15.00 & 17 & 85.00 & 1 & 50.00 & 1 & 50.00 \\
\hline Amoxicillin/clavulanate & 20 & 90.91 & 2 & 9.09 & 9 & 45.00 & 11 & 55.00 & 2 & 100.00 & 0 & 0.00 \\
\hline Cloxacillin & 19 & 86.36 & 3 & 13.64 & 19 & 95.00 & 1 & 5.00 & 1 & 50.00 & 1 & 50.00 \\
\hline Cefotaxime & 19 & 86.36 & 3 & 13.64 & 12 & 60.00 & 8 & 40.00 & 1 & 50.00 & 1 & 50.00 \\
\hline Clindamycin & 19 & 86.36 & 3 & 13.64 & 19 & 95.00 & 1 & 5.00 & 2 & 100.00 & 0 & 0.00 \\
\hline Ciprofloxacin & 17 & 77.27 & 5 & 22.73 & 18 & 90.00 & 2 & 10.00 & 1 & 50.00 & 1 & 50.00 \\
\hline Vankomycin & 17 & 77.27 & 5 & 22.73 & 14 & 70.00 & 6 & 30.00 & 2 & 100.00 & 0 & 0.00 \\
\hline Methicillin & 17 & 77.27 & 5 & 22.73 & 20 & 100.00 & 0 & 0.00 & 2 & 100.00 & 0 & 0.00 \\
\hline Levofloxacin & 15 & 68.18 & 7 & 31.82 & 18 & 90.00 & 2 & 10.00 & 1 & 50.00 & 1 & 50.00 \\
\hline Amoxicillin & 14 & 63.64 & 8 & 36.36 & 3 & 15.00 & 17 & 85.00 & 1 & 50.00 & 1 & 50.00 \\
\hline Ceftazidime & 14 & 63.64 & 8 & 36.36 & 4 & 20.00 & 16 & 80.00 & 1 & 50.00 & 1 & 50.00 \\
\hline Sulfamethoxazole/trimethoprim & 11 & 50.00 & 11 & 50.00 & 17 & 85.00 & 3 & 15.00 & 1 & 50.00 & 1 & 50.00 \\
\hline Gentamicin & 8 & 36.36 & 14 & 63.64 & 10 & 50.00 & 10 & 50.00 & 2 & 100.00 & 0 & 0.00 \\
\hline Tetracycline & 3 & 13.64 & 19 & 86.36 & 4 & 20.00 & 16 & 80.00 & 1 & 50.00 & 1 & 50.00 \\
\hline Amikacin & 2 & 9.09 & 20 & 90.91 & 11 & 55.00 & 9 & 45.00 & 1 & 50.00 & 1 & 50.00 \\
\hline Ampicillin & 2 & 9.09 & 20 & 90.91 & 2 & 10.00 & 18 & 90.00 & 1 & 50.00 & 1 & 50.00 \\
\hline Cephradine & 0 & 0.00 & 22 & 100.00 & 0 & 0.00 & 20 & 100.00 & 0 & 0.00 & 2 & 100.00 \\
\hline Doxycycline & 0 & 0.00 & 22 & 100.00 & 2 & 10.00 & 18 & 90.00 & 1 & 50.00 & 1 & 50.00 \\
\hline Kanamycin & 0 & 0.00 & 22 & 100.00 & 0 & 0.00 & 20 & 100.00 & 0 & 0.00 & 2 & 100.00 \\
\hline Erythromycin & 0 & 0.00 & 22 & 100.00 & 1 & 5.00 & 19 & 95.00 & 1 & 50.00 & 1 & 50.00 \\
\hline Azithromycin & 0 & 0.00 & 22 & 100.00 & 3 & 15.00 & 17 & 85.00 & 0 & 0.00 & 2 & 100.00 \\
\hline
\end{tabular}

Table 5: Antimicrobial susceptibility tests of K. pneumoniae

\begin{tabular}{|c|c|c|c|c|c|c|}
\hline \multirow[b]{3}{*}{ Antimicrobial } & \multicolumn{6}{|c|}{ Number $(n=1)$} \\
\hline & \multicolumn{2}{|c|}{ Sensitive } & \multicolumn{2}{|c|}{ Intermediate } & \multicolumn{2}{|c|}{ Resistant } \\
\hline & $\mathrm{n}$ & $\%$ & $\mathrm{n}$ & $\%$ & $\mathrm{n}$ & $\%$ \\
\hline Ampicillin/sulbactam & 1 & 100.00 & 0 & 0.00 & 0 & 0.00 \\
\hline Cefazolin & 1 & 100.00 & 0 & 0.00 & 0 & 0.00 \\
\hline Ceftriaxone & 1 & 100.00 & 0 & 0.00 & 0 & 0.00 \\
\hline Ceftazidime & 1 & 100.00 & 0 & 0.00 & 0 & 0.00 \\
\hline Aztreonam & 1 & 100.00 & 0 & 0.00 & 0 & 0.00 \\
\hline Cefepime & 1 & 100.00 & 0 & 0.00 & 0 & 0.00 \\
\hline Ertapenem & 1 & 100.00 & 0 & 0.00 & 0 & 0.00 \\
\hline Meropenem & 1 & 100.00 & 0 & 0.00 & 0 & 0.00 \\
\hline Amikacin & 1 & 100.00 & 0 & 0.00 & 0 & 0.00 \\
\hline Gentamicin & 1 & 100.00 & 0 & 0.00 & 0 & 0.00 \\
\hline Ciprofloxacin & 1 & 100.00 & 0 & 0.00 & 0 & 0.00 \\
\hline Tigecycline & 1 & 100.00 & 0 & 0.00 & 0 & 0.00 \\
\hline Sulfamethoxazole/trimethoprim & 1 & 100.00 & 0 & 0.00 & 0 & 0.00 \\
\hline Nitrofurantoin & 0 & 0.00 & 1 & 100.00 & 0 & 0.00 \\
\hline Ampicillin & 0 & 0.00 & 0 & 0.00 & 1 & 100.00 \\
\hline
\end{tabular}


Table 6: Sensitivity of GABHS and S. aureus againts Oral Antimicrobials

\begin{tabular}{|c|c|c|c|c|c|c|}
\hline \multirow[b]{2}{*}{ Antimicrobial } & \multicolumn{2}{|c|}{ GABHS (n=22) } & \multicolumn{2}{|c|}{ S. aureus $(\mathrm{n}=20)$} & \multicolumn{2}{|c|}{ S. epidermidis $(\mathrm{n}=2)$} \\
\hline & $\mathrm{n}$ & $\%$ & $\mathrm{n}$ & $\%$ & $\mathrm{n}$ & $\%$ \\
\hline Clindamycin & 19 & 86.36 & 19 & 95.00 & 2 & 100.00 \\
\hline Ciprofloxacin & 17 & 77.27 & 18 & 90.00 & 1 & 50.00 \\
\hline Levofloxacin & 15 & 68.18 & 18 & 90.00 & 1 & 50.00 \\
\hline Chloramphenicol & 21 & 95.45 & 3 & 15.00 & 1 & 50.00 \\
\hline Amoxicillin/clavulanate & 20 & 90.91 & 9 & 45.00 & 2 & 100.00 \\
\hline Sulfamethoxazole/trimethoprim & 11 & 50.00 & 17 & 85.00 & 1 & 50.00 \\
\hline Amoxicillin & 14 & 63.64 & 3 & 15.00 & 1 & 50.00 \\
\hline Tetracycline & 3 & 13.64 & 4 & 20.00 & 1 & 50.00 \\
\hline Ampicillin & 2 & 9.09 & 2 & 10.00 & 1 & 50.00 \\
\hline Azithromycin & 0 & 0.00 & 3 & 15.00 & 0 & 0.00 \\
\hline Doxycyclin & 0 & 0.00 & 2 & 10.00 & 1 & 50.00 \\
\hline Erythromycin & 0 & 0.00 & 1 & 5.00 & 1 & 50.00 \\
\hline
\end{tabular}

In this study, there was only one sample recovered $K$. pneumoniae and it was resistant to ampicillin (sensitivity $0.00 \%$ ) (Table 5). Sensitivity of GABHS, S. aureus and S. epidermidis against various oral antimicrobials were presented in Table 6. Clindamycin was an oral antimicrobial which had a sensitivity of more than $85.00 \%$ in all three bacteria.

\section{Discussion}

In this study, GABHS and $S$. aureus were the most common isolated microorganisms from secondary infection in scabies, with GABHS slightly more common than $S$. aureus. The occurance was similiar to the observations made by Currie and Carapetis (2000). Bacterial infection following scabies might be related to the deterioration of skin barrier as the outcome from repetitive scratching. An analysis of the skin lipid showed inhibitory effect of linolenic acid, an essential free fatty acid normally present on intact skin which is responsible for inhibition of Staphylococcus colonization and a potential bactericidal factor against Streptococcus pyogenes as well as against bacteria which are normally present in skin microflora (Bergsson et al., 2001). In addition, Streptococcus and Staphylococcus have the main components of adhesin that will bind to fibronectin, an adhesin receptor in the host. In non-intact skin, fibronectin binds easily to bacteria, allowing the bacterial colonization to occur on the skin (Craft, 2012).

Our study showed among all tested specimens, $S$. epidermidis accounted for $4.44 \%$. This organism is a component of the normal cutaneous flora (Otto, 2009). However, if the number increases it can be an opportunistic pathogen (Otto, 2009).

Most pyodermas are caused by Gram positive bacteria (Craft, 2012), though it can be caused by Gram negative bacteria, such as Pseudomonas aeruginosa $(P$. aeruginosa), Proteus vulgaris (P. vulgaris), Escherichia coli (E. coli) and K. pneumonie (Kakar et al., 1999). K. pneumoniae is one of the normal flora of the skin which is found most often in leg and buttock lesions (Grice and Segre, 2011; Pinto-Almeida et al., 2012). It is suspected that the most probable sources of these organisms are the rectal and vaginal orifices, where they normally reside (Brook, 1995). K. pneumoniae was isolated in the present study in the co-infection with GABHS (2.94\%). The other study by Brook (1995) have been reported $3.33 \%$ mixed infections due to GABHS and $K$. pneumoniae in scabies with secondary infection.

In the present study, the sensitivity of various bacteria to amoxicillin/clavulanate is varied, as the drug was widely used in the community. Besides as a treatment of bacterial infections on the skin, amoxicillin/clavulanate is also often prescribed by other clinicians to treat lower respiratory tract infections, urinary tract infections, otitis media, etc (Lammie and Hughes, 2016).

The sensitivity of GABHS and $S$. aureus to cloxacillin in our study was more than $86.36 \%$, while only $50.00 \%$ sensitivity to $S$. epidermidis. All of the Gram possitive cocci in this study had a low sensitivity to amoxicillin and ampicillin (less than 63.64\%). Likewise, the high resistance to these antimicrobials in the present study is in agreement with some other reports (Klevens et al., 2007; Seybold et al., 2006). This could also be due to indiscriminate usage of this antimicrobials in the community and the emerged of penicillinase producing strains (Gandhi et al., 2012). Group A betahemolytic Streptococcus, S. aureus and K. pneumoniae had high sensitivity to ciprofloxacin (greater than $77.27 \%$ ) in this study, whereas sensitivity to levofloxacin was only high in S. aureus $(90.00 \%)$. This study shows similar result to those earlier study which found the sensitivity of GABHS and $S$. aureus to ciprofloxacin more than $70.00 \%$ (El Kholy et al., 2003). The high sensitivity of fluoroquinolone in children due to their limited used, since this class of drugs should not be given if less than 18 years old (Choi et al., 2013). Group A beta-hemolytic Streptococcus, S. aureus and S. epidermidis have high 
sensitivity to clindamycin (more than $85.00 \%$ ) in this present study. Increased sensitivity of Gram-positive bacteria to clindamycin probably due to the decreasing in the prescription of this antibiotic for various diseases (Lewis and Jorgensen, 2005).

In this study, the most sensitive drugs for secondary bacterial infections in scabies caused by GABHS, $S$. aureus, S. epidermidis and $K$. pneumoniae were carbapenem class, because the drug is not widely used in the community. However, this drug was only available for intravenous administration, therefore it is not a treatment option for uncomplicated pyodermas.

Based on this study, antimicrobials that could be given for infections caused by GABHS and/or $S$. aureus were cloxacillin, clindamycin, ciprofloxacin, ceftriaxone and methicillin. However, only the first three antimicrobials mentioned were available in oral form in Indonesia. Clindamycin and cloxacilin can be used either for pediatric and adults (Chen et al., 2011), unfortunately cloxacillin was not always available in many regions in Indonesia. Whereas ciprofloxacin can not be used for children because it may cause arthropathy (Choi et al., 2013), tendon disorders and cartilage changes (Kim et al., 2001).

According to our study result, antimicrobials choice for skin infection caused by $S$. epidermidis were amoxicillin/clavulanate, methicillin, clindamycin, gentamicin and vancomycin, however only amoxicillin/clavulanate and clindamycin were available in oral form. Antibiotics that could be given orally and still sensitive to $K$. pneumoniae were ciprofloxacin and sulfamethoxazole-trimethoprim, while other sensitive drugs only available intravenously, such as ampicillinsulbactam, cefazolin, ceftriaxone, ceftazidime, cefepime, amikacin, gentamicin, aztreonam, ertapenem, meropenem and tigecyclin.

The sensitivity of GABHS and $S$. aureus as the most common causes of secondary infection in scabies were low to amoxicillin, ampicillin, erythromycin, azithromycin, doxycycline, tetracycline, cephradine, amikacin, gentamicin, kanamycin and ceftazidime. This may be due to the widespread use of the drugs, without proper indication, inadequate dosage, long-term use and emerged of strains bacteria resistant to antimicrobials.

\section{Conclusion}

Oral clindamycin is a drug of choice which can be given to pediatric or adults patients for the treatment of secondary bacterial infection following scabies. The use of antimicrobials rationally can alter bacterial susceptibility patterns, resulting in changes of resistance pattern to antimicrobials. Therefore, antimicrobial susceptibility testing should be revised periodically.

\section{Acknowledgment}

This study was supported by Universitas Padjajaran, Bandung, West Java, Indonesia. (Grant No. 855/UN6.3.1/PL/2017).

\section{Author's Contributions}

Hendra Gunawan, Unwati Sugiri and Nurhasanah: All experiments.

Kristina Makarti: Manuscript preparation.

Oki Suwarsa: Design the research plan and organized the study.

\section{Conflict of Interest Statement}

We declare that we have no conflict of interest

\section{Ethics}

The study was approved by the Health Research Ethics Committee, Faculty of Medicine, Universitas Padjadjaran, Bandung, West Java, Indonesia with ethical clearance.

\section{References}

Bergsson, G., J. Arnfinnsson, Ó. SteingrÍmsson and H. Thormar, 2001. Killing of Gram-positive cocci by fatty acids and monoglycerides. APMIS, 109: 670-678. DOI: 10.1034/j.1600-0463.2001.d01-131.X

Bowen, A.C., A. Mahé, R.J. Hay, R.M. Andrews and A.C. Steer et al., 2015. The global epidemiology of impetigo: A systematic review of the population prevalence of impetigo and pyoderma. PloS One 10: e0136789-e0136789.

DOI: 10.1371/journal.pone.0136789

Brook, I., 1995. Microbiology of secondary bacterial infection in scabies lesions. J. Clin. Microbiol., 33: 2139-2140.

Chen, A.E., K.C. Carroll, M. Diener-West, T. Ross and J. Ordun et al., 2011. Randomized controlled trial of cephalexin versus clindamycin for uncomplicated pediatric skin infections. Pediatrics, 127: e573-e580. DOI: $10.1542 /$ peds.2010-2053

Choi, S.H., E.Y. Kim and Y.J. Kim, 2013. Systemic use of fluoroquinolone in children. Korean J. Pediatr, 56: 196-201. DOI: 10.3345/kjp.2013.56.5.196

CDCP, 2013. Antibiotic resistance threats in the United States. Centers for Disease Control and Prevention, US Department of Health and Human Services.

Craft, N., 2012. General Considerations of Bacterial Diseases. In: Fitzpatrick's Dermatology in General Medicine. Goldsmith, L.A., B.A. Gilchrest, A.S. Paller, D.J. Leffell and K. Wolff (Eds.), Mc Graw-Hill, New York, pp: 2121-2147. 
Currie, B.J. and J.R. Carapetis, 2000. Skin infections and infestations in Aboriginal communities in northern Australia. Australas J. Dermatol., 41: 139-143. DOI: 10.1046/j.1440-0960.2000.00417.x

Edison, L., A. Beaudoin, L. Goh, C.E. Introcaso and D. Martin et al., 2015. Scabies and bacterial superinfection among American Samoan children, 2011-2012. PloS One, 10: e0139336-e0139336. DOI: 10.1371 /journal.pone.0139336

El Kholy, A., H. Baseem, G.S. Hall, G.W. Procop and D.L. Longworth, 2003. Antimicrobial resistance in Cairo, Egypt 1999-2000: A survey of five hospitals. J. Antimicrob. Chemother., 51: 625-630. PMID: 12615864

Gandhi, S., A. Ojha and N.K.P. Ranjan, 2012. Clinical and bacteriological aspects of pyoderma. N Am. J. Med. Sci., 4: 492-492. DOI: $10.4103 / 1947-2714.101997$

Grice, E.A. and J.A. Segre, 2011. The skin microbiome. Nat. Rev. Microbiol., 9: 244-244. DOI: $10.1038 /$ nrmicro 2537

Halpern, A.V. and W.R. Heymann, 2008. Bacterial Disease. In: Dermatology, Bolognia, J.L. and R.P. Rapini, Mosby Elsevier, New York, pp: 1075-1106.

Hay, R.J., N.E. Johns, H.C. Williams, I.W. Bolliger and R.P. Dellavalle et al., 2014. The global burden of skin disease in 2010: An analysis of the prevalence and impact of skin conditions. J. Invest. Dermatol., 134: 1527-1534. DOI: 10.1038/jid.2013.446

Kakar, N., V. Kumar, G. Mehta, R.C. Sharma and R.V. Koranne, 1999. Clinico-bacteriological study of pyodermas in children. J. Dermatol., 26: 288-293.

Kim, S.M.B., G.K. Kim and J.Q. Rosso, 2001. Systemic Antibacterial Agents. Comprehensive Dermatologic Drug Therapy. W.B. Saunders, Philadelphia,

Klevens, R.M., M.A. Morrison, J. Nadle, S. Petit and K. Gershman et al., 2007. Invasive methicillin-resistant Staphylococcus aureus infections in the United States. JAMA, 298: 1763-1771.

DOI: $10.1001 /$ jama.298.15.1763

Lammie, S.L. and J.M. Hughes, 2016. Antimicrobial resistance, food safety and one health: The need for convergence. Annu. Rev. Food Sci. Technol., 7: 287-312.

DOI: 10.1146/annurev-food-041715-033251

Lewis, J.S. and J.H. Jorgensen, 2005. Inducible clindamycin resistance in staphylococci: Should clinicians and microbiologists be concerned? Clin. Infect. Dis., 40: 280-285. DOI: 10.1086/426894
Otto, M., 2009. Staphylococcus epidermidis-the "accidental" pathogen. Nat. Rev. Microbiol., 7: 555-555. DOI: 10.1038/nrmicro2182

Pincus, D.H., 2006. Microbial identification using the bioMerieux VITEK ${ }^{\circledR} 2$ System. Encyclopedia Rapid Microbiol. Meth., 1: 1-32.

Pinto-Almeida, T., A. Rosmaninho, I. Lobo, R. Alves and M. Selores, 2012. Exuberant cutaneous ulcers on the buttocks caused by multi-resistant. Dermatol. Online J. 18: 15-15. PMID: 22948065

Rodriguez-Iturbe, B. and M. Haas, 2016. Post-streptococcal glomerulonephritis. Oklahoma City (OK) University of Oklahoma Health Sciences Center, University of Oklahoma Health Sciences Center.

Seybold, U., E. Kourbatova, J. Johnson and S. Halvosa, 2006. Emergence of community-associated 529 methicillin-resistant Staphylococcus aureus USA300 genotype as a major 530 cause of health care-associated blood stream infections. Clin. Infect. Dis., 42: 647-656.

Shetty, N., G. Hill and G. Ridgway, 1998. The Vitek analyser for routine bacterial identification and susceptibility testing: protocols, problems and pitfalls. J. Clin. Pathol., 51: 316-323.

Streeton, C., J. Hanna, R. Messer and A. Merianos, 1995. An epidemic of acute post-streptococcal glomerulonephritis among Aboriginal children. Paediatr. Child Health, 31: 245-248. PMID: 7669388

Twele, L., E. Moyen, K. Zhang, B. Dalton and D. Church et al., 2010. Methicillin-resistant Staphylococcus aureus endocarditis and de novo development of daptomycin resistance during therapy. Can. J. Infect. Dis. Med. Microbiol., 21: 89-93. PMID: 21629617

Vanderkoo, O.G., D.B. Gregson, J.D. Kellner and K.B. Laupland, 2011. Staphylococcus aureus bloodstream infections in children: A population-based assessment. Paediatr. Child Health, 16: 276-280.

White, A.V., W.E. Hoy and D.A. McCredie, 2001. Childhood post-streptococcal glomerulonephritis as a risk factor for chronic renal disease in later life. Med. J. Aust., 174: 492-496.

Wong, C.J. and D.L. Stevens, 2013. Serious group A streptococcal infections. Med. Clin. North Am., 97: 721-736. DOI: 10.1016/j.mcna.2013.03.003 